\title{
Une stratégie pour immortaliser des lignées orientées vers l'endoderme, le neuroectoderme ou le mésoderme à partir du tératocarcinome de la souris
}

\author{
MH Buc-Caron 1*, JM Launay 2, PJ Marie 3, O Kellermann 1 \\ 1 Institut Pasteur, 25, rue du docteur Roux, 75724 Paris; \\ 2 Hôpital St-Louis, 1, avenue C Vellefaux, 75010 Paris; \\ ${ }^{3}$ Hôpital Lariboisière, 2, rue Ambroise, 75475 Paris cedex 10, France
}

(15e réunion du groupe Développement INRA, Paris, 24-26 mai 1989)

\begin{abstract}
Résumé - Afin d'immortaliser des cellules fixées à des stades précoces de l'embryogenèse, des plasmides contenant la région précoce de SV40 ont été introduits dans les carcinomes embryonnaires (EC) F9 et 1003. Seule la construction PK4 dans laquelle les oncogènes de SV40 sont placés sous le contrôle du promoteur E1A de l'adénovirus 5, a permis l'expression des oncogènes de SV40 au cours des premiers stades de la différenciation des EC. Des clones correspondant à des précurseurs des lignages neuroectodermique, mésodermique ou endodermique ont alors été sélectionnés avec une fréquence élevée, selon les critères suivants: sélection de cellules immatures :

- ayant perdu les marqueurs des EC,

- ayant un phénotype stable,

- immortalisées par l'expression des oncogènes de SV40,

- capables de différencier in vivo ou in vitro le long d'un seul lignage.

La description de clones correspondant à des précurseurs des différents lignages de l'embryon est donnée à titre d'exemple.
\end{abstract}

tératocarcinome / oncogène / immortalisation / détermination / différenciation

Summary - A strategy to immortalize, from mouse teratocarcinomas, cell lines committed to endoderm, neuroectoderm or mesoderm. With the aim of immortalizing embryonic cells fixed at early embryonic stages, various plasmids carrying the SV40 early region were introduced into the mouse embryonal carcinomas (EC) F9 and 1003. Only the construction PK4, in which the SV40 oncogenes are placed under the control of the adenovirus E1A promoter, led to the immortalization of the cells at the onset of differentiation. Clones corresponding to committed precursors of each embryonic lineage (neuroectoderm, mesoderm and endoderm) were then selected with high efficiency according to the following strategy: selection of immature cells which:

- have lost EC cell markers,

- keep a stable phenotype,

- are immortalized by the expression of the SV4O oncogenes and are still able to differentiate along a restricted lineage in vitro or in vivo.

Examples of an endodermal precursor $(H 7)$ which differentiates into extraembryonic and embryonic endoderm, of a neuroectodermic clone (ICII) committed to a serotoninergic differentiation, and of a mesodermal osteogenic clone $(\mathrm{Cl})$ which gives rise to bone in vivo and in vitro, are given.

teratocarcinoma / oncogene / immortalisation / détermination / differentiation

* Correspondance et tirés à part 


\section{INTRODUCTION}

De nombreuses lignées de cellules multipotentielles ont été établies in vitro à partir de tératocarcinomes (carcinome embryonnaire, EC) (Jakob et Nicolas, 1987) ou directement à partir d'embryons (embryonal stem cell, ES) (Evans et Kaufman, 1981). Ces lignées sont capables in vivo et/ou in vitro de différencier en dérivés des 3 feuillets embryonnaires : neuroectoderme, mésoderme, endoderme.

Mais au cours de leur différenciation, les EC ou les cellules d'embryon perdent la capacité de se diviser et seulement quelques lignées permettant principalement d'étudier des mécanismes de différenciation terminale ont pu être établies à partir des tératocarcinomes (Nicolas et al, 1976). Les expériences de mutagénèse (Boon et al, 1975) ou de transformation virale (Knowles et al, 1980) visant à isoler, à partir des EC multipotentielles ou à partir d'embryons, des cellules souches restreintes dans leurs potentialités à un seul lignage de l'embryon, se sont avérées négatives. Notre objectif fut donc de construire des plasmides susceptibles de faire exprimer des oncogènes dans les premiers stades de la différenciation cellulaire afin d'immortaliser in vitro des cellules souches «déterminées" vers 1 des 3 feuillets embryonnaires. Une cellule déterminée est une cellule engagée dans une voie de différenciation, qui n'exprime pas encore les marqueurs des cellules différenciées, et qui est capable de différencier plus avant sous l'effet d'inducteurs spécifiques. De telles lignées, issues du tératocarcinome ou de l'embryon permettraient d'analyser les événements impliqués dans les mécanismes de détermination et de différenciation cellulaires.

\section{MATÉRIEL ET MÉTHODES}

\section{Culture et différenciation des cellules}

Les cellules sont cultivées sur des boîtes traitées pour la culture de tissu dans du milieu DMEM (Dulbecco's modified Eagle's medium) contenant $10 \%$ de sérum foetal de veau (FBS). Les clones ont été obtenus par dilution limite $(0,3$ cellule par puits). Pour induire leur différenciation, les cellules $1 \mathrm{C} 11\left(2 \times 10^{3}\right.$ cellules par $\mathrm{cm}^{3}$ ) sont ensemencées en présence de $10^{-3}$ mol/l dibutyryl cyclic AMP (dbcAMP) et de $0,05 \%$ acide carboxylique cyclohexane (CCA). Pour induire la minéralisation in vitro, les cellules $C_{1}$ sont cultivées en agrégats sur des boîtes de Petri non traitées pour la culture. Après 5-8 j, les agrégats sont induits à différencier en présence de $1 \%$ FBS, d'acide ascorbique $(50 \mu \mathrm{g} / \mathrm{ml})$ et de $\beta$-glycérophosphate $(7$ $\mathrm{mmol} /$ ). Les nodules calcifiés apparaissent rapidement $(2 \mathrm{j})$ à partir des agrégats.

\section{Immunocytochimie}

Les marqueurs spécifiques ont été détectés par immunofluorescence indirecte en utilisant plusieurs antisérums : l'anticorps monoclonal PAB419 détectant l'antigène T de SV40, les anticorps anti-LCAM et NCAM, antifibronectine et anticytokératine endo A (Kellermann et al, 1987), les anticorps monoclonaux de rat : SY38 antisynaptophysine (Dr Wiedenmann, Heidelberg, RFA), YC5/45 antisérotonine (Dr Milstein, Cambridge, UK) l'anticorps de lapin anti Metenképhaline (Dr Cesselin, Paris). Des seconds anticorps purifiés par affinité, couplés à la Rhodamine, ont permis de visualiser les sites de fixation du premier anticorps. Des contrôles négatifs sans le premier anticorps ont été effectués dans toutes les expériences.

\section{Métabolisme de la sérotonine}

La biosynthèse de sérotonine a été mesurée à partir du tryptophane par radiochromatographie 
et par dosage radioenzymatique. Les paramètres de la capture de sérotonine sont mesurés à $37^{\circ} \mathrm{C}$ après lavage des cellules dans un milieu sans sérum. La capture a été inhibée par les drogues suivantes: clomipramine $\left(10^{-8} \mathrm{~mol} / /\right)$, R04-1284 (10-5 mol/ $)$; paroxetine $\left(10^{-8} \mathrm{~mol} / \mathrm{h}\right)$; ouabaïne $\left(10^{-4} \mathrm{~mol} / \mathrm{l}\right)$.

\section{Identification histochimique de la phosphatase alcaline}

Les coupes de nodules calcifiés (5 $\mu \mathrm{m}$ d'épaisseur) sont fixés à $4^{\circ} \mathrm{C}$ dans l'éthanol $95 \%$, exposées au naphtol ASBI phosphate (30 min, $37^{\circ} \mathrm{C}, \mathrm{pH} 8,6$ ) et observées après contrecoloration par l'hématoxyline.

\section{RÉSULTATS \\ Construction de plasmides recombinants adéno-SV40}

L'expression d'un gène viral dépend des fonctions cellulaires de l'hôte. Les cellules multipotentielles de l'embryon ou les cellules EC sont résistantes à l'infection par de nombreux virus comme polyome, SV40 ou les rétrovirus. SV40 ne s'exprime pas avant le $9^{e}$ ou le $10^{e}$ jour de l'embryogénèse de la souris et, in vitro, seules les cellules différenciées permettent l'expression virale et sont alors transformées. En revanche, l'adénovirus s'exprime aussi bien dans les cellules EC que dans les cellules différenciées (Kelly et Condamine, 1982).

Nous avons donc décidé de faire appel aux régions promotrices activatrices de l'adénovirus pour faire exprimer la région précoce du virus SV40 dans les cellules embryonnaires précoces. Un plasmide, PK4, a été construit avec la région précoce de SV40 en aval de la séquence promotrice activatrice du gène $\mathrm{E} 1 \mathrm{~A}$ de l'adénovirus de type 5 . Des plasmides dérivés de
PK4 portant la région activatrice de SV40, soit à l'intérieur du promoteur E1A, soit en aval de la région précoce de SV40, ont également été fabriqués (fig 1 ).

\section{Expression de PK4 dans les cellules de carcinome embryonnaire}

Des lignées EC, F9 (Bernstine et al, 1973) et 1003 (McBurney, 1976), ayant intégré le plasmide PK4, ont été obtenues par cotransfection de ce plasmide avec le plas-

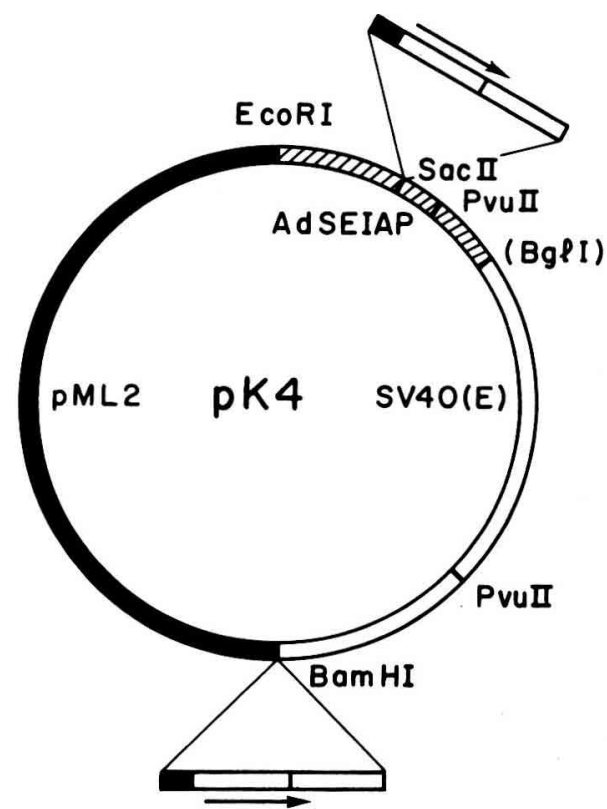

Fig 1. Plasmides PK4 et dérivés. PK4 contient la région précoce de SV40 (blanc) liée à la région promotrice activatrice de l'adénovirus 5 (hachurée) dans le vecteur PML2 (noir) (Kellermann et Kelly, 1986). Les dérivés PK4 ${ }^{S}$ et PK4 ${ }^{B}$ contiennent la région activatrice de SV40 insérée, respectivement, au site Sacl/ du promoteur E1A (nucléotide 360) et au site BamH1 (à la liajson SV40/PML2). La région activatrice de SV40 contient la répétition des 72 paires de bases (nucléotides 128-270) de SV40 (blanc) et une région adjacente (nucléotides 115-128) (noir). 
mide PSVtkneo $\beta$ et sélection des clones résistants au G418. La permissivité de ces 2 lignées vis-à-vis de l'expression de PK4 s'est révélée très différente. Le promoteur E1A de l'adénovirus permet une expression des fonctions précoces de SV40 dans les clones 1003 PK4 à l'état EC. L'expression de l'antigène $T$ de $S V 40$ révélée par immunofluorescence indirecte n'est donc pas létale pour les cellules EC, n'induit pas leur différenciation et n'empêche pas les cellules 1003-PK4 de différencier, après induction in vitro ou in vivo, en dérivés des 3 feuillets embryonnaires.

En revanche, le promoteur E1A de l'adénovirus n'est pas suffisant pour permettre une expression de l'antigène $T$ de SV40 dans les clones F9 transformés. Cette restriction n'est levée, ni par une augmentation du nombre de copies intégrées, ni par l'adjonction de l'activateur de SV40 dans le plasmide PK4 en amont ou en aval des gènes précoces de SV40 (tableau I). Par contre l'induction de la différenciation des clones F9PK4 par l'acide rétinoïque (AR) et le dibutyryl AMPc (dbAMPc) s'accompagne d'une expression de l'antigène $T$ de SV40 (Kellermann et Kelly, 1986).
Stratégie pour sélectionner des clones précurseurs des lignages cellulaires à partir des clones F9 K4b2 et 1003 PK4

L'induction de la différenciation des lignées EC ayant intégré dans leur génome PK4 (F9 K4B2, 1003-PK4) conduit à l'obtention d'une population immortalisée tumorigène exprimant l'antigène T de SV40. Cette population contient à la fois des dérivés du neuroectoderme, du mésoderme et de l'endoderme ainsi que des cellules immatures. Supposant que les cellules immatures situées au voisinage de cellules différenciées pouvaient correspondre aux précurseurs recherchés, nous avons sélectionné, puis cloné les précurseurs potentiels sur la base des critères suivants :

- perte des marqueurs des EC, absence de réversion vers l'état $E C$;

- expression de l'antigène T de SV40 et immortalisation;

- phénotype stable à l'état de précurseur et différenciation reproductible vers un seul lignage.

L'expression de l'antigène T de SV40 nous a permis d'obtenir des clones avec une fréquence élevée (efficacité de clo-

Tableau l. Expression de l'antigène T de SV40 dans les lignées EC transformées par PK1 (Gluzman et Ahrens, 1982), PK4 et ses dérivés. "Le nombre de plasmides intégrés selon les clones a été estimé par comparaison avec la lignée SVT2. ** L'antigène T de SV40 s'exprime après induction de la différenciation.

$\begin{array}{llcc}\text { Promoteur } & \begin{array}{l}\text { Activateur } \\ \text { de SV40 }\end{array} & \begin{array}{c}\text { Copies } \\ \text { intégrées }\end{array}\end{array} \quad \begin{gathered}\text { Clones EC expriment T } \\ \text { nombre de clones transformés }\end{gathered}$

\begin{tabular}{lllll}
\hline F9PK1 & SV40 & + & 2 à 5 & $0 / 3$ \\
F9PK4 & E1a & + & 2 à 5 & $0 / 5$ \\
F9PK4 & E1a & + & 2 à 5 & $0 / 3$ \\
F9PK4 & E1a & - & 2 à 50 & $0 / 5^{* *}$ \\
1003 PK4 & E1a & - & 1 à 3 & $3 / 3$ \\
\hline
\end{tabular}


nage $>80 \%$ ). En revanche, la sélection de lignées de précurseurs s'est révélée particulièrement difficile du fait de l'absence de marqueurs connus spécifiques des étapes précoces de l'embryogénèse. L'identité des clones n'a pu être établie qu'après induction, grâce à l'éventail des différenciations obtenues (fig 2). Nous disposons maintenant de clones représentatifs de cellules souches de differrentes voies de différenciation (Kellermann et al, 1987).

\section{Caractérisation de clones précurseurs des voles endodermique, neuroectodermique ou mésodermique}

Tous les clones immortalisés expriment l'antigène T de SV40, présentent un phénotype stable à l'état de précurseur et sont capables de se différencier in vitro et/ou in vivo selon un programme cohérent et reproductible. A titre d'exemple, voici les propriétés de 3 de ces clones.

\section{Précurseurs endodermiques}

Le clone F9 K4b2 a été induit à se différencier en agrégats en présence d'AR et de dbcAMP. Les cellules qui se différencient à la périphérie des agrégats expriment l'antigène T de SV40. Elles ont le phénotype des cellules extra-embryonnaires d'endoderme viscéral, elles synthétisent l' $\alpha$ fœtoprotéine (Hogan et al, 1981) et la villine (Buc-Caron et al, 1989). Des clones endodermiques immortalisés ont été sélectionnés après remise en culture des cellules bordant les agrégats. In vitro, le clone H7 se différencie en cellules d'endoderme viscéral. In vivo, $\mathrm{H} 7$ a les propriétés d'un précurseur endodermique multipotentiel. Les tumeurs induites par l'injection du clone $\mathrm{H} 7$ à des souris syngéniques sont constituées d'un mélange de dérivés endodermiques extra-embryonnaires (endoderme pariétal et viscéral) et embryonnaires (épithélium intestinal avec des cellules à mucus) (Kellermann et al, 1987).


Fig 2. Représentation schématique du mode d'obtention et de sélection des précurseurs de lignages. 


\section{Précurseur neuroectodermique}

En absence d'inducteur, les cellules ICII ont un phénotype épithélial (fig 3A) expriment la cytokératine endo $A$ et la protéine d'adhésion $L$ CAM. En présence de dbAMPc, plus de $95 \%$ des cellules (ICII*) développent des extensions bipolaires (fig 3C) et continuent de se diviser et d'exprimer l'antigène $T$ de SV40. Elles expriment la protéine d'adhésion NCAM, la fibronectine, la synaptophysine (marqueur des vésicules synaptiques) et la Metenképhaline. Par des dosages immunochimiques et radioenzymatiques, nous avons montré que ces cellules ICII synthétisent, stockent (fig 3D) et captent un neuromédiateur, la sérotonine. La capture de sérotonine par ICll est abolie par des drogues qui inhibent son transport membranaire et granulaire dans les neurones sérotoninergiques.

Les celiules présentent donc 2 états très homogènes: l'état de précurseur ICII et l'état différencié ICII* de phénotype neuronal ou neuroendocrinien sérotoninergique (Buc-Caron et al, 1990).

\section{Précurseur ostéogénique}

Le clone $C_{1}$ a été sélectionné parmi d'autres clones mésoblastiques car il exprime des phosphatases alcalines de type osseux. Les cellules $C_{\mid}$expriment l'antigène $T$ de SV40 et présentent un phénotype stable en absence d'inducteur (fig $3 E)$. In vivo, les tumeurs induites par l'injection du clone $C_{I}$ sont des ostéosar- comes. In vitro, en présence de $\boldsymbol{\beta}$ glycérophosphate et d'acide ascorbique, $\mathrm{C}_{\mid}$forme des nodules calcifiés (fig 3F). Les clichés de microscopie électronique confirment la minéralisation sur les fibres de collagène.

$\mathrm{C}_{1}$ est donc un clone mésoblastique ostéogénique, capable de former de l'os in vitro et in vivo (Kellermann et al, 1990).

\section{CONCLUSION}

Le promoteur E1A de l'adénovirus permet l'expression des oncogènes de SV40 au cours des premiers stades de la différenciation des cellules EC. Ceci nous a permis d'immortaliser, de cloner, et de sélectionner des lignées correspondant à des cellules souches orientées vers les voies neuroectodermique, mésodermique et endodermique. Ces clones immortalisés sont des outils cellulaires permettant d'étudier les mécanismes de régulation génétique et épigénique des cellules embryonnaires précoces.

\section{RÉFÉRENCES}

Bernstine EG, Hooper ML, Grandchamp S, Ephrussi B (1973) Alkaline phosphatase activity in mouse teratoma. Proc Natl Acad Sci USA 70, 3899-3903

Boon T, Kellermann O, Mathy E, Gaillard JA (1975) Mutagenized clones of a pluripotent teratoma cell line: variants with decreased differentiation or tumor formation ability. In: Teratoma and Differentiation. Academic Press, New York, 161-166

Fig 3. Cellules ICII : (A) phase, (B) Marquage nucléaire de l'antigène T de SV40 par immunofluorescence indirecte. Fixation methanol. Cellules ICI $I^{*}$ : (C) phase, (D) Marquage par des anticorps antisérotonine. Fixation paraformaldéhyde $3 \%$ puis éthanol $70 \%$. Cellules $\mathrm{C}_{1}:(\mathrm{E})$ phase d'une culture exponentielle (F) Marquage sur coupe par des anticorps antiphosphatase alcaline d'un agrégat calcifié. 
Buc-Caron MH, Lamblin D, Kellermann O (1989) $\mathrm{Fg}$ teratocarcinoma aggregates express villin upon differentiation into visceral endoderm-like cells. Bio Cell 65, 195-198

Buc-Caron MH, Launay JM, Lamblin D, Kellermann O (1990) Serotonin uptake, storage and synthesis in an immortalized committed cell line derived from mouse teratocarcinoma Proc Natl Acad Sci USA 87, 1922-1926

Evans MJ, Kaufman MH (1981) Establishment in culture of pluripotential cells from mouse embryos. Nature 292, 154-156

Gluzman Y, Ahrens N (1982) SV40 early mutants that are defective for viral synthesis but competant for transformation of cultured rat and Simian cells. Virology 123, 78-92

Hogan BLM, Taylor A, Adamson E (1981) Cell interactions modulate embryonal carcinoma cell differentiation into parietal or visceral endoderm. Nature 291, 235-237

Jakob H, Nicolas JF (1987) Mouse teratocarcinoma cells. In: Methods in Enzymology 151, 66-81

Kellermann O, Kelly F (1986) Immortalization of early embryonic cell derivatives after the transfer of the early region of simian virus 40 into F9 teratocarcinoma cells. Differentiation $32,74-81$
Kellermann O, Buc-Caron MH, Gaillard J (1987) Immortalization of precursors of endodermal, neuroectodermal and mesodermal lineages, following the introduction of the simian virus (SV40) early region into F9 cells. Differentiation 35, 197-205

Kellermann O, Buc-Caron MH, Maris PJ, Lamblin D, Jacob F (1990) An immortalized osteogenic cell line derived form mouse teratocarcinoma is able to mineralize in vivo and in vitro. J Cell Biol 110, 123-132

Kelly F, Condamine $H$ (1982) Tumor viruses and early mouse embryos. Biochim Biophys Acta 651, 105-141

Knowles BB, Pan S, Solter D, Linnenbach A, Croce C, Huebner K (1980) Expression of $\mathrm{H}$ 2 laminin and SV40 T and TASA on differentiation of transformed murine teratocarcinoma cells. Nature 288, 615-618

McBurney M (1976) Clonal lines of teratocarcinoma cells in vitro: differentiation and cytogenetic characteristics. J Cell Physiol 89, 441456

Nicolas JF, Avner P, Gaillard J, Guenet JL, Jakob H, Jacob $F$ (1976) Cell lines derived from teratocarcinomas. Cancer Res 36, 4224-4231 